Revista Universo Contábil, ISSN 1809-3337

FURB, v. 6, n.2, p. 153-169, abr./jun., 2010

doi:10.4270/ruc.2010218

Disponível em www.furb.br/universocontabil

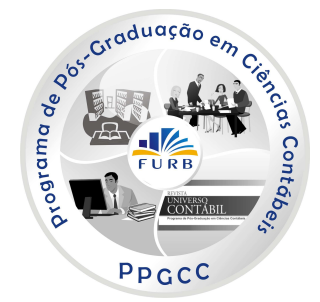

\title{
REVISION DE UNA EXPERIENCIA DE LA ENSEÑANZA DE LA CONTABILIDAD BAJO LA MODALIDAD A DISTANCIA, UTILIZANDO LAS TICs*
}

\section{REVIEW OF A DISTANCE ACCOUNTING TEACHING EXPERIENCE USING ICTS}

\author{
Martín Ernesto Quadro \\ Doctorando en Ciencias Económicas de la FCE/UNC \\ Profesor Adjunto de la Facultad de Ciencias Económicas \\ Universidad Nacional de Córdoba (FCE/UNC) \\ Endereço: Avda. Valparaíso s/n - Ciudad Universitaria \\ CEP: 5000 - Córdoba - Argentina \\ E-mail: quadro@eco.unc.edu.ar \\ Telefone: 00-54-351-4437300
}

Adriana Andreone

Magíster en Investigación Educativa pela FCE/UNC Profesora Asociada de la Facultad de Ciencias Económicas Universidad Nacional de Córdoba (FCE/UNC) Endereço: Avda. Valparaíso s/n - Ciudad Universitaria CEP: 5000 - Córdoba - Argentina

E-mail: alaje@eco.unc.edu.ar Telefone: 00-54-351-4437300

\section{RESUMEN}

Son muchos los avances conseguidos por la implementación de las Tecnologías de la Información y Comunicación (TICs) en el ámbito de la enseñanza universitaria. Las ponderadas bondades de estas herramientas fueron pregonadas a diestra y siniestra por distintos especialistas en la materia, propiciando su uso cada vez mas generalizado. El objetivo del trabajo es analizar los principales aspectos referidos a la implementación de un programa de enseñaza universitaria de Contabilidad en un entorno virtual. Para ello se analizó cómo el uso de las TICs afectó los modos de enseñanza de esta asignatura, las interacciones entre los distintos actores del proceso de enseñanza y aprendizaje, el diseño de materiales. Se trató de una investigación descriptiva e interpretativa, donde se utilizaron metodologías cuantitativas y cualitativas. Con los datos obtenidos se realizaron nexos, cruces y triangulaciones que permitieron la confiabilidad de los mismos. Se utilizó el estudio de casos

\footnotetext{
* Artigo recebido em 25.11.2009. Revisado por pares em 21.12.2009. Reformulado em 29.03.2010. Recomendado para publicação em 31.03.2010 por Ilse Maria Beuren (Editora). Publicado em 30.06.2010. Organização responsável pelo periódico: FURB.
} 
como método de análisis. Para este escrito en particular, se seleccionó el caso testigo del tutor responsable del área Contable, desarrollando cada uno de los ejes de análisis: configuraciones didácticas, interacción, estrategias de apoyo, materiales de enseñanza y la presencia o ausencia de tendencias y controversias entre la educación presencial y la educación a distancia. Se concluye que el uso de las TICs potenciará las buenas prácticas de enseñanza en aquellos casos donde la tecnología puede estar integrada a la propuesta metodológica de manera tal que favorezca la construcción del conocimiento. Por el contrario, el abuso, el uso de las mismas mediante un empelo banal o superficial y que parten de una concepción meramente utilitarista, seguramente producirá efectos totalmente contrarios a los deseados.

Palabras-clave: Enseñanza. Contabilidad. E-learning. TICs.

\section{RESUMO}

A aplicação das Tecnologias de Informação e Comunicação (TICs) no âmbito do ensino universitário tem proporcionado muitos avanços. Os benefícios desses instrumentos foram enaltecidos por diferentes especialistas da área, incentivando o seu uso cada vez mais difundido. $\mathrm{O}$ estudo tem como objetivo analisar os principais aspectos relativos à implementação de um programa de ensino universitário de contabilidade em ambiente virtual. Foi examinado como a utilização das TICs afetou a forma de ensino deste curso, as interações entre os diferentes agentes no processo de ensino e aprendizagem e o desenho dos materiais didáticos. Foi realizada pesquisa descritiva e interpretativa, que utilizou tanto metodologia quantitativa como qualitativa. Com os dados obtidos, realizaram-se conexões, cruzamentos e triangulações que permitiram a confiabilidade dos resultados. Utilizou-se o estudo de caso como um método de análise. Neste trabalho em particular, foi selecionado o relato do coordenador responsável pela área de contabilidade, desenvolvendo cada um dos eixos de análise: o contexto educacional, a interação, as estratégias de apoio, materiais didáticos e a presença ou ausência de tendências e controvérsias entre ensino presencial e ensino à distância. Conclui-se que a utilização das TICs reforçará as boas práticas de ensino nos casos em que a tecnologia possa estar integrada na proposta metodológica de modo a promover a construção do conhecimento. Em contrapartida, o abuso de utilização destas tecnologias através de um emprego banal ou superficial e que se baseia puramente em uma concepção utilitarista, certamente produzirá efeitos completamente contrários aos pretendidos.

Palavras-chave: Ensino. Contabilidade. E-learning. TICs.

\section{ABSTRACT}

The application of Information and Communication Technologies (ICTs) as part of university education has provided many advances. The benefits of these tools have been enhanced by various experts in the area, encouraging their use more widespread. This study aims to analyze the main aspects related to an implementation of a university accounting education program in a virtual environment. It was examined how the use of ICT affected how the course was taught, the interactions between different actors in the process of teaching and learning and the design of educational materials. A descriptive and interpretative research was done, using both quantitative and qualitative methodology. Having these data, connections, intersections and triangulations were performed to allow the reliability of results. A case study was used as a method of analysis. In this work in particular was selected the report of the coordinator in charge of the accounting area, developing each of the axes of analysis: the educational context, interaction, support strategies, teaching materials and the presence or absence of trends and controversies between presential and distance learning. It is concluded 
that the use of ICTs will reinforce good teaching practices where technology can be integrated into the proposed methodology in order to promote the construction of knowledge. In contrast, the abuse of these technologies through a menial or shallow use based purely on a utilitarian conception will certainly lead to completely contrary effect to those desired.

Keywords: Teaching. Accounting. E-learning. ICTs.

\section{INTRODUCCIÓN}

Son muchos los avances conseguidos por la implementación de las Tecnologías de la Comunicación y la Información (TICs) en el ámbito de la enseñanza universitaria. Las ponderadas bondades de estas herramientas fueron ampliamente difundidas por distintos especialistas en la materia, propiciando su uso cada vez más generalizado. Poco a poco, con el objetivo de lograr "la democratización del conocimiento", "la igualdad de oportunidades", "reducir la brecha digital"; las TICs comenzaron a expandirse lentamente pero a paso firme. En investigaciones anteriores (ANDREONE, 2002), se observó cómo su aplicación fue inicialmente potenciada por las ofertas de cursos de actualización y posgrados; para luego bajar a las aulas de grado afianzando su presencia. Como la evolución tecnológica nos brinda cada vez mayor capacidad (de procesamiento, almacenamiento, transmisión) con costos menores; esta expansión fue consolidada también en los programas de educación a distancia.

Al decir de Burbules y Callister (2001), existen condiciones históricas que anteceden y ponen un techo a las elecciones y actividades de docentes y alumnos, construyendo maneras, configuraciones y limitando también las posibilidades de la comunidad, en relación al uso de las TICs. Las propuestas de educación a distancia se presentan en un contexto de comunicación en el que se producen intercambios y construcciones de significados diferentes al obtenido en prácticas educativas presenciales. De esta manera surgen nuevas prácticas en el pensamiento de los profesores, en las teorías implícitas sobre los alumnos y en la construcción de significados en el aula.

En ese sentido, nos preguntamos:

a) ¿Qué tipo de proyectos se generaron a partir del uso de las tecnologías, en la educación a distancia?

b) ¿Qué forma adopta la intervención didáctica en el aula, a partir del uso de las TICs, en los programas de educación a distancia?

c) ¿Es viable la enseñanza a distancia de la contabilidad, en entornos virtuales?

Un antecedente en nuestra Facultad fue el proyecto educativo "Programa para la Enseñanza de la Contabilidad (PECO)", que sentó interesantes precedentes en el campo de la aplicación de las nuevas tecnologías en la enseñanza de la contabilidad. Con el objetivo de enseñarles a los alumnos dentro de un contexto más cercano, no solo desde lo tecnológico sino también desde la perspectiva de su realidad laboral. Para ello, se diseñó un programa informático que permitía "procesar la información contable" con las funciones básicas que el estudiante encontraría en los estudios contables u oficinas cuando egresara.

El programa estaba montado en una plataforma educativa (aula virtual) con servicios tales como foro, chat, email, suscripción a noticias. Cada alumno podía conectarse desde su casa, un cyber o desde las PC de la misma Facultad. Como atractivo adicional, las clases se impartían en comisiones con una cantidad reducida de estudiantes y utilizando PC. La implementación de esta innovadora propuesta, evidenció que las TICs, por sí solas, no necesariamente implican un impacto positivo en los procesos de enseñanza y aprendizaje, es la propuesta educativa en su conjunto.

Asimismo, planteo algunos interrogantes tales como: ¿Son las TICs un contenido a enseñar junto con la contabilidad? ¿Tienen igual jerarquía y peso? ¿Cómo lograr que el 
"medio" no sea más importante que el "mensaje”? ¿Qué importancia debe darse en las evaluaciones al uso de esta herramienta por sobre el conocimiento de la disciplina? Que tipo competencias deben tener los docentes que utilicen las TICS? Su uso, $₫$ asegura la igualdad de acceso?

El presente artículo y sus conclusiones, son fruto de una investigación más amplia que analiza las prácticas de enseñanza en la Facultad de Ciencias Económicas (FCE) de la Universidad Nacional de Córdoba (UNC), a partir del uso de las TICs dentro de un Programa de Educación a Distancia (Una mirada a las Prácticas de la Enseñanza en la Facultad de Ciencias Económicas). En el marco evaluativo de la Secretaría de Ciencia y Técnica de la Universidad Nacional de Córdoba, Años 2006/07, Proyecto de investigación acreditado.

\section{OBJETIVOS}

El objetivo de este trabajo es analizar los principales aspectos referidos a la implementación de la modalidad a distancia para la enseñanza de la Contabilidad, indagando cómo el uso de las TICs afectó los modos de enseñanza de esta asignatura, las interacciones entre los distintos actores del proceso de enseñanza y aprendizaje, el diseño de materiales. Asimismo, se pretende:

a) estudiar el uso del espacio virtual en relación con la capacidad de acceso técnico (cuantitativo) y el uso vinculado a la capacidad de acceso comprensivo (cualitativo) que docentes y estudiantes hacen de la plataforma educativa en la propuesta de enseñanza a distancia;

b) analizar en la comunicación docente/estudiante, la presencia o ausencia de apoyos provisionales pedagógicos, que le permitan al alumno avanzar en su proceso de estudio a distancia;

c) observar la existencia de espacios comunicativos (a través de foros), que permitan mejorar las problemáticas de comprensión de los estudiantes, en el área contable.

\section{METODOLOGÍA}

Se utilizó el estudio de casos como método de análisis. Para este escrito en particular, se seleccionó el caso testigo del tutor responsable del área Contable, desarrollando cada uno de los ejes de análisis: configuraciones didácticas, interacción, estrategias de apoyo, materiales de enseñanza y la presencia o ausencia de tendencias y controversias entre la educación presencial y la educación a distancia.

Se trató de una investigación descriptiva e interpretativa, donde se utilizó metodologías cuantitativas y cualitativas. Con los datos obtenidos se realizaron nexos, cruces y triangulaciones que permitieron la confiabilidad de los mismos.

Entre las principales técnicas utilizadas, podríamos citar: a) entrevistas en profundidad a los docentes / tutores (12 en total); b) encuestas a estudiantes (15\% de la población de cada Cátedra); c) datos secundarios de ficha académica, ficha personal y socio-económica.

\section{ALGUNAS CONSIDERACIONES DIDÁCTICAS}

Por un lado, nos interesa profundizar en nuestro análisis el uso de las TICs en la educación a distancia, considerando el espacio de comunicación virtual, ya que plantea nuevas oportunidades de interactuar entre docentes, estudiantes y contenido de la enseñanza. Por otro lado, nos preocupa develar cuál es la manera de utilizarla en nuestro campo disciplinar (la contabilidad) y en nuestra Institución universitaria, a fin de lograr un favorable manejo de la tecnología en los proyectos educativos.

Reconociendo, al decir de Litwin (2005), que se trata de un camino muy complejo, ya que los docentes incorporan las tecnologías de diferentes maneras y con propósitos 
desparejos: están aquellos que piensan utilizarlas para resolver los problemas de comprensión de los estudiantes, otros que las piensan como un peligro que puede minimizar la comprensión de los estudiantes, además se encuentran aquellos que las utilizan de modo estandarizado, respondiendo a una demanda de la época sin pensar las mismas dentro de su proyecto educativo.

Al mismo tiempo, son los jóvenes los que acceden y se hacen asiduos usuarios y demandantes de las mismas. El uso en los cyber o en sus hogares, introduce nuevas maneras de pensar y comunicarse. Estas experiencias les permiten pensar en red, vivir la hipertextualidad, sostener intereses tecnológicos. Al respecto argumentan Mercer y Gonzáles Estepa (1991, p. 82):

El acceso a la información no es equivalente al acceso al conocimiento y a las oportunidades de educación. Debemos abordar estas nuevas formas de comunicación como estimulantes oportunidades para el uso del lenguaje con el fin de pensar conjuntamente, y como nuevas formas de andamiaje de los procesos de construcción del conocimiento, de los estudiantes en el uso del lenguaje en tanto instrumento del pensamiento.

Se trata entonces de un cambio fuerte y progresivo, para lograr un uso de las TICs al servicio del conocimiento, es decir, vinculadas a la pasión por conocer. En las universidades y en nuestra casa de estudio en particular, hemos observado en investigaciones anteriormente realizadas, que existe una incorporación paulatina de las nuevas tecnologías.

El uso de las TICs se da de manera diferente: según el contenido de enseñanza, si tienen incorporadas en su forma de trabajo profesional, si participan en comunidades virtuales (tales como Institutos de investigación, ONG), si han realizado capacitaciones. Como en toda nueva incorporación tecnológica, se presentan múltiples dificultades, algunas vinculadas a la capacidad tecnológica y otras a la capacidad de acceder en sus usos cotidianos. Si existe un equipamiento tecnológico adecuado, la mayor dificultad se presenta en sus usos cotidianos.

Las ventajas reconocidas para la educación a distancia fue haber permitido un mayor acceso al sistema educativo de aquellas poblaciones que, por diferentes causas, no podían acceder a los sistemas presenciales. El impacto de las nuevas tecnologías presagia un uso masivo, es probable que muchas personas accedan al conocimiento a través de ellas. De allí, se deriva la fuerza de la virtualidad. En el marco estrictamente pedagógico, hay que observar cómo se articulan las prácticas presenciales con estos nuevos entornos de campus, donde se instalan materiales, actividades, foros, es decir, cómo se complementan unas y otras.

\section{CONTEXTO INSTITUCIONAL: EL CICLO BÁSICO A DISTANCIA EN LA FCE DE LA UNC}

Un estudio realizado por Goldenhersh et al. (2006), referido a la deserción en la Facultad de Ciencias Económicas (FCE) de la Universidad Nacional de Córdoba (UNC); permitió realizar el siguiente análisis con relación a la cohorte de alumnos 1997: a) el 25\% del total de ingresantes deserta en el primer año de cursado; b) el $11 \%$ del total de ingresantes deserta en el segundo año de cursado; e c) durante los 5 años restantes, desertaron a razón del $5 \%$ anual.

Con estos datos se infiere que de los alumnos que ingresan a la Facultad, aproximadamente el $61 \%$ deserta en alguna instancia de la carrera (las autoras explican que ese alto porcentaje de deserción esta influido por estudiantes fantasmas, que nunca realizaron actividad académica alguna, y por estudiantes que se inscriben en dos o más carreras al mismo tiempo). El porcentaje restante se distribuye entre los que se gradúan efectivamente $(9 \%)$, están próximo a hacerlo (14\%) o tienen un atraso intermedio (16\%). 
En el citado estudio, al preguntar a los alumnos sobre las distintas causales de la deserción se destacan principalmente: a) no saber estudiar; b) problemas económicos; c) problemas con las evaluaciones. Entre las restantes causas mencionadas, se encuentran: a) no habituarse a la vida universitaria; b) poco contacto con los docentes; c) falta de vocación; d) disconformidad con los métodos pedagógicos.

Las autoras proponen salir de la institución en jaque a la institución en juego, en el sentido de comprometer a la Facultad al diseño e implementación de políticas académicas activas que permitan abordar el problema de la deserción desde una perspectiva integral revisando el papel y las prácticas docentes en el Profesorado Universitario. Es en este contexto que se desarrolla el proyecto de dictado de todas las asignaturas del Ciclo Básico bajo la modalidad a distancia.

\subsection{El contexto curricular}

En la actualidad, la estructura curricular del plan de estudio se compone de tres ciclos: Ciclo de Nivelación, Ciclo Básico y Ciclo Profesional. En el Ciclo de Nivelación (es un espacio curricular clave para entender las disciplinas económicas, su proyección en el futuro profesional. También, con lo que significa ser estudiante universitario), los alumnos comienzan a trabajar por primera vez con la metodología de enseñanza semipresencial. En el Ciclo Básico, comienza la trama de las diferentes áreas y materias que forman parte del Plan de Estudios. El objetivo de este Ciclo es avanzar en los fundamentos y herramientas básicas y generales de la matemática, la contabilidad, la economía, la administración y el derecho, que son comunes para las diferentes carreras que se cursan en la FCE. En el ultimo tramo curricular, el Ciclo Profesional, los estudiantes se especializan en los contenidos de la carrera elegida (Contador Público, Licenciatura en Economía y Licenciatura en Administración).

El programa de Ciclo Básico a Distancia, propone el dictado de todas las asignaturas que lo componen bajo esa modalidad. Para nuestro análisis focal seleccionamos la asignatura Sistemas de Información Contable II bajo la modalidad a distancia, que integra dicho ciclo curricular.

Como hemos sostenido en investigaciones anteriores (Plataformas educativas en Internet: condicionantes tecnológicos culturales. Proyecto acreditado SeCyT UNC 2003), el uso de las tecnologías no tiene un efecto neutro ya que se encuentra relacionado con una propuesta de enseñanza que presupone algunos paradigmas por sobre los cuales está cimentada. Asimismo, las tradiciones, cultura y la impronta de la institución, establecen en forma implícita o explícita reglas macro, que a su vez condicionan las propuestas o proyectos educativos que se emprenden.

Nos interesa reflexionar sobre los propósitos que motivaron la necesidad de establecer el dictado de una materia de contenido contable, bajo la modalidad a distancia. Para ello es necesario analizar el contexto imperante al momento de sancionarse la reglamentación que da nacimiento al proyecto.

\subsection{La enseñanza de la Contabilidad a distancia}

En general, una propuesta de educación a distancia, implica las siguientes prácticas por parte de los estudiantes:

a) trabajar autónomamente a partir de las orientaciones que encontrarán en las guías de estudio;

b) realizar una lectura sistemática del material de estudio y resolver las actividades propuestas, según un cronograma sugerido de tareas;

c) conformar grupos para compartir el proceso de estudio; 
d) asistir a las tutorías presenciales optativas en la medida de las necesidades y posibilidades. En dichos encuentros podrán tomar contacto con el enfoque que los docentes dan a los distintos temas; consultar a los mismos sobre dudas y dificultades en la comprensión, encontrarse con compañeros, realizar trabajos cooperativos, acercarse a la dinámica de la vida universitaria en el espacio institucional.

Asimismo, dentro de la propuesta a distancia los alumnos pueden ingresar a un espacio o aula virtual montada en la Web (llamada plataforma educativa) que les permite reemplazar la presencialidad del aprendizaje. En general, las aulas virtuales como Moodle, Educativa, ofrecen las siguientes funcionalidades:

a) disponer de información complementaria al material impreso en distintos formatos (video, audio);

b) encontrar los correos electrónicos de profesores y compañeros para comunicarse;

c) relacionar los contenidos desde la hipertextualidad;

d) el aprendizaje y la enseñanza se dan en instancias asincrónicas, a diferencia de la modalidad presencial;

e) Participar con los profesores y compañeros a través de foros.

Para el dictado de la asignatura Sistemas de Información Contable II bajo esta modalidad, se dispone de los siguientes recursos:

a) equipo docente - está conformado por un profesor titular a cargo y tres profesores asistentes;

b) aula virtual - la Facultad cuenta con la plataforma E-ducativa que contiene los servicios de e-learning tales como chats, foros, mails;

c) bibliografía y material didáctico desarrollado especialmente para esta modalidad;

d) tutorías presenciales - la materia se dicta en forma semestral. Los estudiantes sólo disponen de cuatro encuentros presenciales en dicho período. Todo el proceso de enseñanza se realiza en el aula virtual.

A continuación, presentaremos cómo se utilizan estas herramientas y las estrategias didácticas aplicadas por el equipo docente de Sistemas de Información Contable II y analizaremos sus implicancias en torno a las prácticas significativas de enseñanza.

\subsubsection{Las tutorías presenciales}

En este tipo de proyectos, el Tutor se concibe como un mediador, que realiza sus intervenciones con el propósito de favorecer la construcción del conocimiento. Es aquél que valora el aprendizaje colaborativo como una forma de socializar y compartir el conocimiento para lograr una construcción superadora. Coincidimos con Maggio (2006) al desatacar que un buen tutor debe saber cuál es el alcance y sentido de las distintas intervenciones didácticas que promueve.

En el caso de la materia que estamos analizando, se encuentran previstas cuatro tutorías presenciales - no obligatorias; a lo largo del semestre. La primera de ellas, está destinada a la presentación de la materia, y las tres restantes al abordaje de problemas que se presentan para el estudio de los diferentes contenidos. Es importante resaltar que la mayor parte de la enseñanza y el aprendizaje se realizan en el aula virtual de la asignatura. Por lo tanto, estas tutorías sólo están pensadas para resolver problemas de comprensión específicos que el estudiante no ha podido resolver en el espacio virtual.

Nos preguntamos, ¿qué importancia tiene la primera tutoría de presentación de la materia, dentro de la propuesta de enseñaza? Indagando al tutor, éste responde: 
temáticos del programa, cómo se regulariza, cuál es el sistema de evaluación, el cronograma de actividades, bibliografía, etc. [...] todo igual que una cátedra presencial. La gran diferencia radica en que también debemos destinar una parte muy importante a explicarles cómo será la metodología de la materia a distancia. Rescatamos la importancia de que autogestionen sus tiempos, presentamos el aula virtual y explicamos cómo se trabaja con ella, etc. Es una tutoría muy importante, ya que sólo nos veremos cara a cara en otras tres tutorías más en todo el semestre. Pero lamentablemente, sólo asisten un $30 \%$ de los alumnos inscriptos

Reflexionando sobre los problemas que ésto ocasiona, el docente acota:

Si bien esta materia es, obviamente a distancia, consideramos muy importante que el alumno asista a esta tutoría de presentación. De lo contrario, al ingresar después en el aula virtual, están muy perdidos [...] o no saben dónde buscar la información o nos ha pasado que ni siquiera saben cómo entrar al sitio. Pierden mucho tiempo ubicándose en la virtualidad. Sabemos que eso les produce algo de desorientación o angustia, y muchos de esos alumnos terminan dejando de cursar esas dos primeras semanas.

En este primer encuentro, el docente recupera las prácticas presenciales de los alumnos, para abordar desde lo ya conocido hacia lo nuevo a conocer, esto es básico en la metodología a distancia. Es decir, el tutor centra sus esfuerzo en establecer nexos o puentes que unan la historia académica de los estudiantes; construida con prácticas desde la presencialidad hacia la virtualidad. Parafraseando a Ausubel (1976), lo ya conocido es central para lo nuevo a conocer.

Analizando las estadísticas de la población de alumnos de la Cátedra (Informe de Ciclo Nivelación 2005 Departamento de Educación a Distancia de la Facultad de Ciencias Económicas UNC), con relación a su participación, observamos que en el primer semestre, de 256 alumnos, sólo tuvieron actuación académica aproximadamente el $40 \%$ (117 estudiantes), en tanto para el re-dictado del segundo semestre, de 572 alumnos, el 48\% (274 alumnos) estuvieron activos académicamente.

Quizás parte de este desgranamiento en el cursado, tenga que ver con lo manifestado por el tutor, referido a cierta desorientación que siente el alumno al cursar una materia con una modalidad distinta a la tradicional (presencial). Por otra parte, la falta de manejo de la herramienta tecnológica tiene asimismo un impacto negativo en el cursado de los alumnos. Es decir, la adopción de nuevas tecnologías crea nuevos problemas: se modifica lo que queremos, intentamos y consideramos posible hacer.

\subsubsection{El aula virtual}

Las TICs constituyen un entorno, un ciberespacio donde se producen interacciones humanas. En relación con este espacio, en el caso particular de nuestro estudio, el intercambio se consolida a través de la plataforma educativa denominada e-ducativa. Aquí se genera el intercambio a través de las diferentes secciones que tiene el diseño de la misma. Queremos resaltar la importancia del Foro de discusión y el correo electrónico como las herramientas de mayor capacidad de interacción. (el chat no está diseñado).

¿En qué medida el aula virtual es utilizada por el docente para incentivar el pensamiento activo del estudiante?

La misma enseñanza se va transformando, el sistema de estudio se modifica, cambian los propósitos que se esperan en torno al aprendizaje de determinados temas o áreas. En ese sentido, el tutor manifiesta al respecto:

El aula tiene muchas secciones, nosotros tratamos de incentivar mucho el uso del Foro por sobre otras secciones. Es más, las consultas de tipo informativo como 
horarios de parciales, aulas en donde se rinden, etc., están totalmente separadas de las consultas de contenido. No respondemos consultas individuales por correo electrónico. Les pedimos a los alumnos que toda duda que tengan sea publicada en el Foro. Esto tiene la ventaja de incentivar la participación, se trabaja sobre la jerarquía de los conceptos, y las relaciones entre ellos, pero también es cierto que hay alumnos que no se animan a publicar una duda por temor a que parezca tonta. Es como cuando estás en el aula y no te animás a preguntar por temor a hacer el ridículo. Acá en la plataforma, queda publicado tu nombre y hay alumnos que tienen miedo de exponerse.

Analizando lo dicho por el tutor, vemos cómo él se interesa por la participación de sus alumnos en los foros, para asegurarse un intercambio que favorezca el aprendizaje desde la enseñanza. Al examinar los accesos a la plataforma (aula virtual de la asignatura, www.edudis.eco.unc.edu.ar), inferimos que existe una participación cuantitativa significativa en aquellas secciones que implican un mayor intercambio entre docentes y alumnos. Como puede observarse en el Cuadro 1, la participación más frecuente se manifiesta en aquellas secciones que exigen una participación activa del docente y estudiante, a través del Foro (52 $\%$ ). En importancia, le sigue, aquellas secciones tales como: noticias (26\%), inicio (8\%), archivos $(5 \%)$, calendario (2\%), presentación (1\%); donde la participación del tutor y del alumno está restringida a un rol de transmisión de información.

\begin{tabular}{l|l|l}
$\begin{array}{l}\text { Secciones } \\
\text { Administración }\end{array}$ & $0-0 \%$ & \\
\hline Presentación & $128-1 \%$ & \\
\hline Asignaturas & $51-0 \%$ & $3315-26 \%$ \\
\hline Noticias & $257-2 \%$ & \\
\hline Calendario & $761-5 \%$ & $6744-52 \%$ \\
\hline Archivos & $151-1 \%$ & \\
\hline Sitios & $225-1 \%$ & \\
\hline Foros & $83-0 \%$ & \\
\hline Preguntas Frecuentes & $1021-8 \%$ & \\
\hline Contactos & $0-0 \%$ & \\
\hline Inicio & $5-0 \%$ & \\
\hline Mis datos & & \\
\hline Búsqueda &
\end{tabular}

\section{Cuadro 1 - Cantidad de accesos a las secciones del aula virtual}

Ahora bien, de estas cifras nos interesa realizar una mirada cualitativa de la comunicación entre estudiantes y tutores; teniendo en cuenta el tipo o clase de intervenciones que tienen los alumnos y tutores en este espacio virtual. Ya que cantidad no puede ser sinónimo de calidad (Cuadro 2).

Este Cuadro 2 nos presenta las cantidades de veces que los estudiantes acceden a las diferentes secciones de la plataforma educativa. Los accesos pueden ser de lectura o de carga. El primer tipo de acceso, implica que el estudiante sólo lee información previamente publicada por sus pares y/o por el tutor. En cambio, los accesos de carga evidencian una actitud más proactiva por parte del alumno ya que se refieren a accesos donde él ha iniciado un nuevo debate en el Foro, o ha respondido a un debate ya abierto o intervenido a través del Correo Electrónico. 


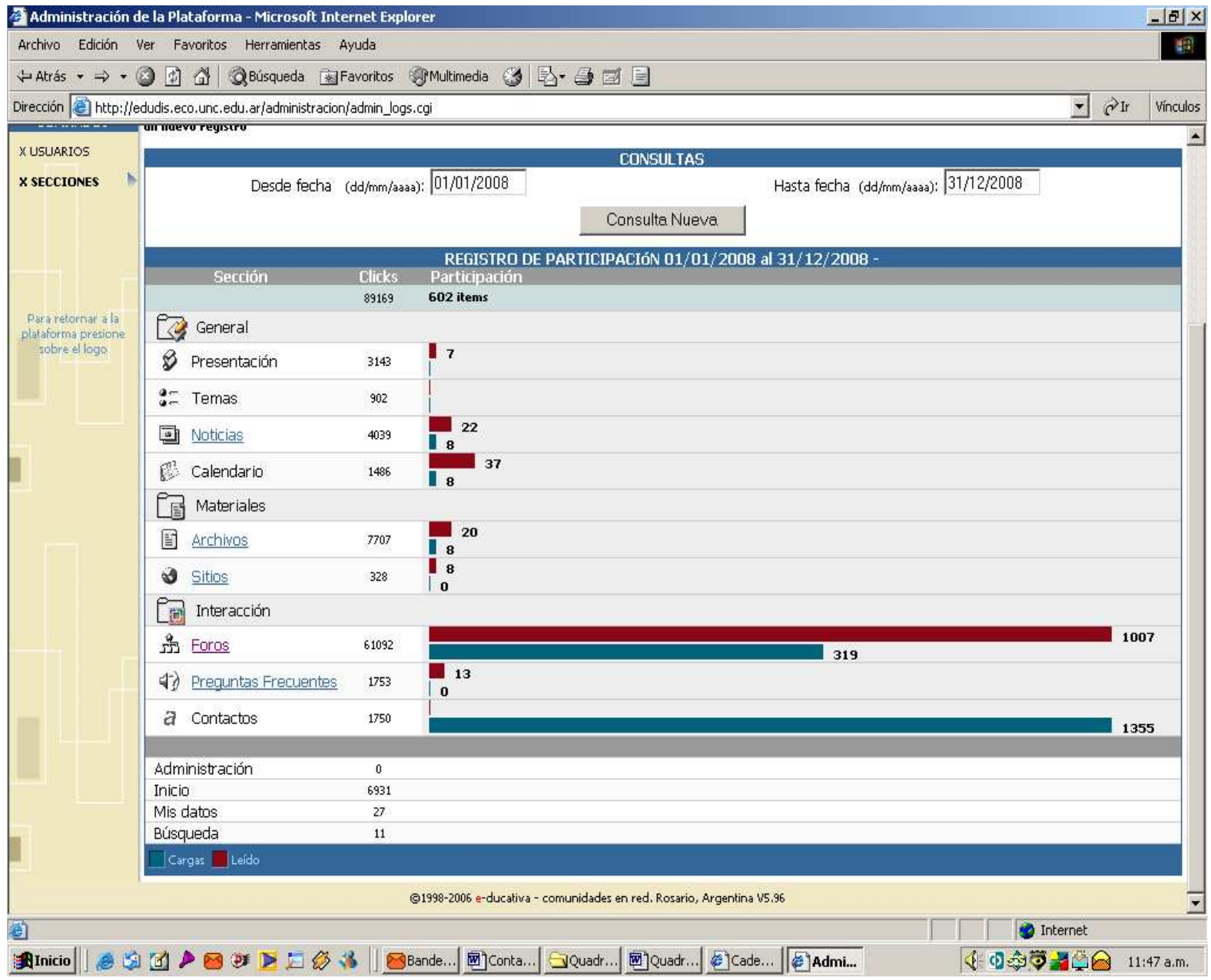

Cuadro 2 - Tipos de acceso a las secciones del aula virtual

Analizando estas estadísticas de acceso, podemos inferir que:

a) las secciones con mayor participación activa del alumno (cargas) son las secciones de: Contactos (les permite comunicarse entre pares y con profesores vía mail) y Foros;

b) en el Foro se destaca como una sección donde la intervención que privilegia el alumno es la lectura (1007 por lectura de foros versus 319 intervenciones por consultas);

c) otras secciones, tales como Calendario, Archivos y Noticias se destacan en menor medida por la participación (por lectura) de los alumnos.

Por lo tanto, es válido afirmar que de todas las secciones que ofrece esta herramienta, los docentes y alumnos privilegian aquellas que permiten el mayor grado de interacción, los tipos de intervención nos revelan interacciones que siguen un patrón tradicional de enseñanza presencial: son mayores los alumnos que no participan de aquellos que sí lo hacen.

\subsubsection{Los Foros}

Esta materia tiene organizados los Foros de manera tal que, para cada capítulo del material de estudio, se encuentra habilitada en la plataforma un Foro para que el alumno pueda plantear sus dudas. De esa manera, como el material tiene 14 capítulos, actualmente se encuentran habilitados 14 foros de contenido. También existe un foro de preguntas de organización, donde el alumno puede preguntar sobre cuestiones administrativas (por ejemplo: cambio de fechas de las evaluaciones) o de información general de la asignatura. 
Es en esta sección, donde el tutor y los alumnos interactúan entre sí con el contenido. Como vimos en el Cuadro 2, a diferencia de la sección de contactos, en el Foro se presentan tipos de acceso pasivos (lectura) o activos (carga). Con respecto a aquellas intervenciones en donde el alumno es quien toma la iniciativa y pregunta, creemos necesario indagar sobre la clase de preguntas se realizan. Estas intervenciones, ¿son sobre el contenido? O más bien, ¿son consultas de tipos administrativas o informativas?

En ese sentido, el Cuadro 3 nos brinda información al respecto. En este último cuadro, podemos observar que una proporción importante de alumnos publican sus consultas en el Foro de Preguntas de Organización, por sobre las preguntas de los foros o capítulos de contenido. También se destaca que en algunos foros, no existe ni intervención del alumno ni de los tutores.

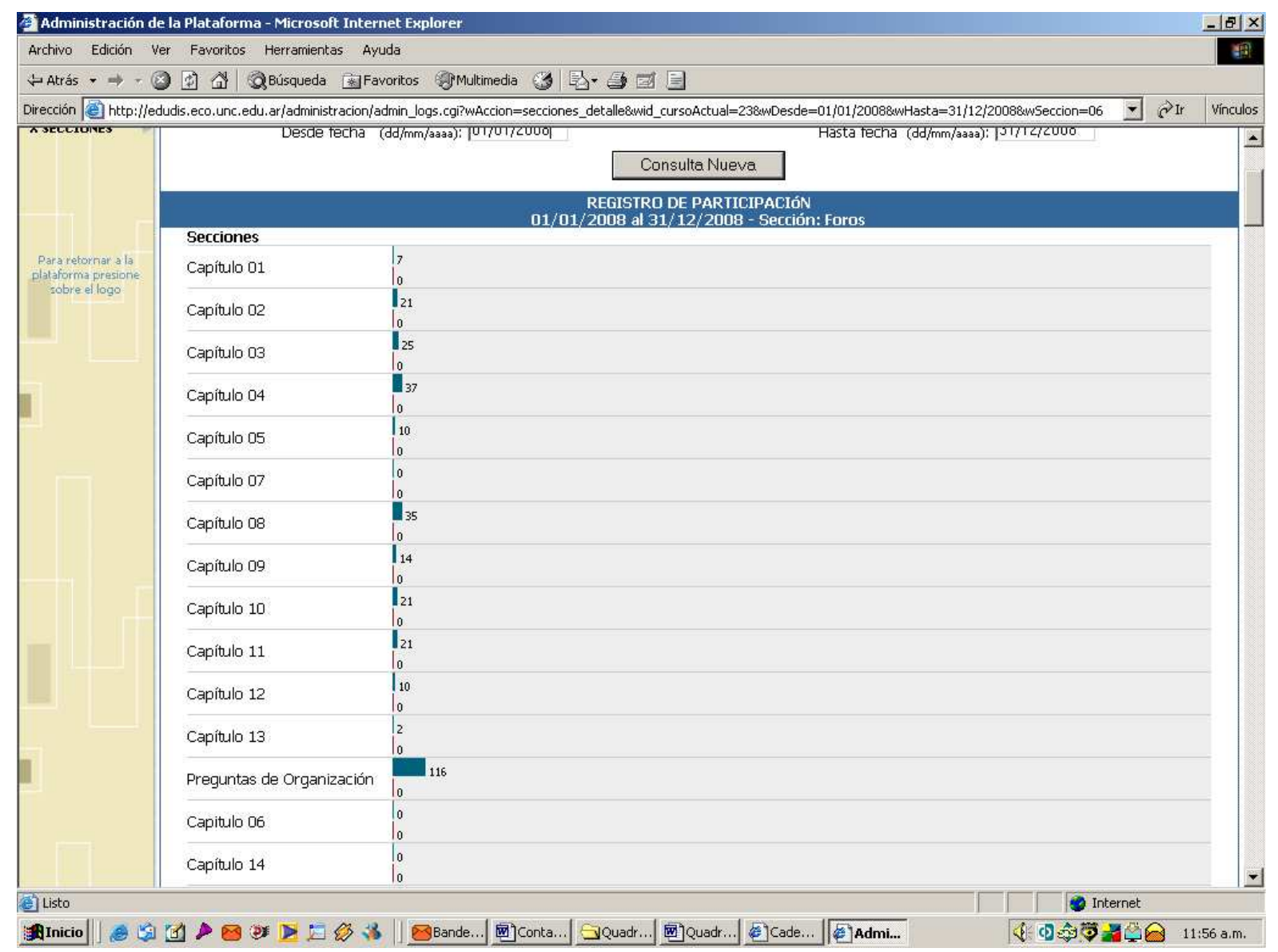

Cuadro 3 - Cantidad de accesos a los Foros por categorías

En relación a los diferentes tipos de intervenciones de los alumnos en estos foros, el docente manifiesta:

Normalmente los alumnos hacen preguntas referidas a cosas que no han entendido del material o piden más ejercitación o una explicación diferente de conceptos desarrollados en el libro. Por supuesto que también están aquellos que hacen una pregunta y te das cuenta de que no han leído o lo que preguntan es algo más básico y de tipo informativo. Pero en general, las preguntas son buenas, en el sentido de que evidencian un análisis y estudio del material detrás de esa pregunta.

Con respecto al papel que cumplen los docentes ante esas intervenciones, el docente complementa: 
Tratamos de no dar la respuesta tan servida [...] la idea es que el alumno vaya descubriendo la respuesta por sí solo, o que otro alumno lo ayude, eso es bueno. La idea es que no sea un dialogo exclusivo entre el docente y el alumno que pregunta, sino que todos puedan participar. Pero no siempre se puede hacer esto o a veces la pregunta es muy concreta y respondemos nosotros.

Algunas intervenciones (reproducidas del aula virtual de la materia) dan cuenta de lo manifestado por el docente.

Intervención 1:

Profesor, no me queda claro la diferencia entre ejercicio contable irregular y otro regular (PABLO)

Me parece que está, hay un ejemplo en la Guía al final del capítulo que te lo aclara mejor (LUCAS).

Me perece una buena idea lo que te plantea tu compañero. ¿Por qué no miras ese ejercicio? Es el número 5 de las actividades de comprensión. De todas formas, te doy una pista. La diferencia está relacionada con el tiempo (DOCENTE).

El alumno responde al día siguiente:

Listo, todo aclarado (PABLO).

Nuevamente el docente interviene:

Me alegra que lo puedas repensar. ¿Te animas a escribir cuál es la diferencia? De esa forma, no sólo podrías compartir tu duda con tus compañeros sino que te serviría para reforzar lo que comprendiste, ¿estás de acuerdo? (DOCENTE).

Si Profesor. La diferencia es que un ejercicio contable regular es cuando en la contabilidad se analizan períodos todos iguales, por ejemplo 12 meses. En cambio un ejercicio contable irregular es cuando el balance se refiere a un período que es menor al habitual. Eso sería cuando la empresa cierra siempre balances anuales y empezó sus actividades un 5 de agosto. Ese primer ejercicio contable es irregular porque es menor a los 12 meses de su actividad. Es así, ¿, no? (PABLO).

Muy bien!. El análisis que hiciste es acertado. Gracias por aclarar esas diferencias (DOCENTE).

\section{Intervención 2:}

Hola!!! No entiendo cómo se realiza la conciliación bancaria, no sé exactamente partiendo desde el saldo banco para llegar al de la empresa qué debo sumar y qué restar (ANA).

Mira, yo te lo digo. Si partís del saldo final de la empresa y:

+ cheques empresa y no banco

- cheque banco y no empresa

- depósito no banco y si empresa

+ depósito banco y no empresa

- gastos bancarios

+ cheques registrados de +

- cheques registrados de -

+ depósitos registrados de -

- depósitos registrados de +

y así llegas al saldo del banco, recuerda que empiezas del último saldo que arroja la empresa (VALERIA)

¡Bien Valeria! ¡Has ayudado a tus compañeros! De esa manera nos ayudamos entre todos! También es importante recordar que en estos esquemas simplificados, lo más 
importante es razonar las operaciones que pueden existir entre el banco y la empresa que deben ser conciliados. De lo contrario caeremos en la memorización. Si buscan en el práctico 7 y 8 van a encontrar situaciones que no están en ese esquema, ¿se animan a resolverlas y darnos algunos ejemplo? (DOCENTE).

Profesor, ¿Pueden ser las transferencias entre cuentas corrientes? (ANA)

Yo creo que sí, también el depósito acreditado pero no realizado por la empresa. ¿Es correcto? (VALERIA).

$\mathrm{Si}$, son correctos ambos ejemplos (DOCENTE).

En estas intervenciones vemos que, el docente como tutor, busca reafirmar como positivo la intervención de otros alumnos en la búsqueda de la respuesta. En estas y otras intervenciones similares analizadas se observa reiteradamente que el tutor insta a que las respuestas sean contestadas con la intervención de otros pares. Asimismo, las intervenciones buscan brindarle una guía al estudiante para que encuentre la respuesta. Este tipo de intervenciones permiten destacar la importancia de una concepción de la enseñanza desde una visión constructivista, que rescate la comprensión por sobre la transmisión.

Por otra parte, al valorar la participación de otros estudiantes se destacan y valoran las instancias de aprendizaje colaborativo. Una de las dificultades de la enseñanza en entornos virtuales es la dificultad para desarrollar lazos o vínculos interpersonales con compañeros; muchas veces la soledad de la virtualidad, atenta contra la continuidad del cursado. Toda actividad (trabajos en grupo, participaciones en chat grupales) que tiendan a entablar y reforzar esos lazos, atenuará ese sentimiento de estar estudiando solo y permite establecer vínculos de pertenencia.

En nuestras observaciones, hemos encontrado que estas estrategias están pensadas y utilizadas para estimular la participación de los estudiantes, pero esto no siempre sucede. Por ejemplo, el foro se plantea como un espacio de diálogo abierto, y no siempre es así, ya que en algunas oportunidades opera como un elemento de exclusión, ausencia y silencio, participando en la lectura del mismo sin realizar preguntas, probablemente debido a la exposición a juicios de valor, tanto de los docentes como del grupo de pares, generando aversión y prevención en los modos de participación. Esto constituye indirectamente un factor que podríamos denominar, junto con Burbules y Callister (2001), limitación al acceso. Ello deberá tenerse en cuenta a la hora de diseñar las estrategias de intervención por parte de los docentes, ya que de lo contrario, se convierte en un criterio de exclusión que opera automáticamente.

\subsection{Material de estudio}

Los materiales de estudio representan una parte distintiva del diseño a distancia o presencial. Históricamente, ocupan un lugar prioritario en la educación y en la cultura escolar. El contenido a enseñar es parte de la tríada didáctica (Enseñanza-Aprendiz-Contenido) y requiere ser presentado y comunicado a los alumnos. Son herramientas materiales y simbólicas que se construyen con el fin de facilitar los procesos de compresión de los alumnos.

En este programa, observamos que son los tutores los responsables de elaborar los materiales virtuales e impresos. Es decir, se logra un producto elaborado principalmente por el profesorado. Son materiales que no suelen responder a criterios económicos del mercado editorial, ni a demandas administrativas. Se centran en la experticia profesional del docente.

Otro aspecto a analizar es el referido al material bibliográfico que se utiliza en la asignatura. La bibliografía se compone de un libro de texto tradicional, utilizado en las demás cátedras de enseñanza presencial y adicionalmente se diseñó un texto especial que sirve de apoyo específicamente para la modalidad a distancia. 
Al respecto el tutor comenta:

Como las tutorías de consultas presenciales, son sólo tres en todo el semestre y los alumnos tardan en familiarizarse con el aula virtual, se pensó en escribir un material o una guía que reemplazara la voz del docente al dar la clase sobre un tema de la materia. Participamos todos los docentes de la Cátedra, más una especialista del Departamento de Educación a Distancia. El libro de textos tiene quince capítulos, entonces nosotros escribimos también quince capítulos de la guía donde, al igual que en una clase, hacemos un resumen de lo principales aspectos o presentamos otros aspectos o formas de encarar el tema, resolvemos ejercicios de maneras alternativas, etc. Fue un trabajo muy arduo, pero a la Cátedra le dejó mucho [...] es más muchas de las estrategias que usamos para escribir ese material lo estamos aplicando ahora para escribir textos en cátedras presenciales.

\section{ACERCA DE LA ENSEÑANZA DE LA CONTABILIDAD}

La enseñanza de la contabilidad, desde lo epistemológico, históricamente se abordó desde un enfoque sistémico al considerar el sistema contable como parte integrante del sistema de información de un ente. Es común encontrar en los libros de textos de contabilidad, un diagrama donde se la identifica con un proceso de transformar datos en información. Esa forma de entender a la contabilidad como disciplina, tiene sus incidencias a la hora de pensar en cómo enseñar sus contenidos.

Bajo esa perspectiva, al momento de diseñar los programas, es habitual encontrar profesores que se esmeran en que el alumno sea un procesador contable, es decir, haga un sinnúmero de asientos, saque cuentas, determine saldos y arme balances. Es frecuente observar en los programas de contabilidad un marcado énfasis en cómo armar la información contable descuidando los fundamentos conceptuales sobre los cuales se sustentan las prácticas. En ese sentido, rescatamos las palabras de Rojas Rojas (2008, p. 269) al expresar que:

\footnotetext{
la educación contable tradicional parte de asignaturas de contabilidad donde se instruye en el marco conceptual de la contabilidad financiera, pero no se enseñan los referentes que permiten comprender la raíz conceptual que da origen a la técnica de registro de los activos, de los pasivos y del patrimonio. Cada vez más compruebo que los estudiantes de últimos semestres saben contabilizar, pero desconocen los problemas éticos que nacen de la valoración de los activos y del patrimonio como meras representaciones financieras de las empresas. Siento que los programas de estudio tal como se encuentran diseñados hoy no han logrado articular proyectos educativos que permitan que los estudiantes detecten la arquitectura del pensamiento contable y las racionalidades contextuales que determinan la vigencia o no, de los conceptos que viabilizan las formas de valoración y de información del patrimonio económico de las empresas.
}

Actualmente, en la reformas de planes de estudio, existe un fuerte sentido crítico al enfoque tradicional, y se están produciendo cambios que destacan la necesidad de resaltar la Teoría Contable por sobre el procedimiento en sí mismo de la información contable. Diversos autores, tales como Tua Pereda (1995), Silva Palavecinos (2003), Seltzer (2004), han manifestado sobre la necesidad de encontrar métodos eficaces para la enseñanza de la contabilidad. Vale decir, se busca establecer algunos criterios para repensar la práctica docente a los efectos de definir una didáctica de la contabilidad en el ámbito universitario. A decir de Seltzer $(2004$, p. 2) se pretende "romper la tradicional antipatía que genera esta disciplina, derivada más de su didáctica que de sus contenidos".

Al ingresar a la problemática de la enseñanza (en nuestro caso de la contabilidad), al decir de Jackson (2002) podemos pensarla como una relación dicotómica entre la enseñanza mimética en contraposición con una transformadora. Bajo la concepción mimética, el 
conocimiento es poseído por el docente quien se encarga de transferirlo al estudiante; consecuentemente las actividades y estrategias docentes están diseñadas para reproducir el conocimiento. El alumno es un recipiente vacío en el cual se depositan los contenidos, sin que ello lo cambie, altere o transforme. La concepción mimética se encuentra frecuentemente en el campo de la enseñanza contable, que traduce una mirada de pensamiento lineal de la contabilidad.

Contrariamente, una enseñanza transformadora es aquella destinada a realizar un cambio cualitativo en el estudiante. Es la buena enseñanza versus la enseñanza exitosa. Va más allá del contenido en sí mismo, se espera que el conocimiento permita un desarrollo en la estructura cognitiva, asumiendo el docente un rol superador, priorizando la construcción del conocimiento por sobre la transmisión. También pone en juego valores, dilemas morales, principios éticos, invita a tomar actitudes críticas frente a lo aprendido. Es decir, este último enfoque analiza a la empresa, su contexto y al interior de la misma, sus las prácticas contables de una manera integral y dinámica.

¿Cuándo una materia es dictada bajo la modalidad a distancia, el uso de las TICs necesariamente favorece el desarrollo de una enseñanza transformadora en vez de una mimética? En realidad, son los proyectos educativos, sus propósitos, sus alcances los que determinan el papel de la tecnología. Un buen docente que desarrolla estrategias que lo acercan a construcciones y maneras críticas de asumir su quehacer didáctico (LITWIN, 1997), será lo que favorezca el aprendizaje; seguramente potenciará sus efectos mediante el uso de las nuevas tecnologías. En ese sentido, coincidimos con Litwin (2005) cuando sostiene que las tecnologías "no son neutras ni pueden separar su carácter de herramienta y entorno de los fines con los que se utilizan".

La configuración didáctica es el fruto de varios factores tales como la concepción de enseñanza, el posicionamiento frente a la disciplina, los criterios usados para la selección de los contenidos, etc. Todos esos factores tienen como único fin lograr un buen aprendizaje de los estudiantes. Las TICs integradas a la propuesta académica tienden a volverse invisibles, porque están y se construyen a partir del proyecto educativo, posibilitando (o buscando) centralmente la construcción de conocimientos compartidos.

Al respecto, Litwin (2005) reconoce tres diferentes usos de la tecnología si consideramos conjuntamente al papel del docente, la concepción del sujeto de aprendizaje y el sentido con el que se entiende el contenido a enseñar. Los usos alternativos pueden ser:

a) la tecnología como una herramienta de actualización de información - es cuando el docente tiene un papel de proveedor de información, siendo el estudiante un mero consumidor. Los docentes tienden a enseñar desde un punto de vista mimético;

b) la tecnología como medio para acceder a contenidos adicionales - normalmente se trata de docentes que las utilizan para complementar los materiales de sus clases;

c) la tecnología como una forma de brindar múltiples opciones, caminos o puertas de entrada al conocimiento.

En nuestra investigación, se observó cómo el tutor de la cátedra estudiada se vale de ciertas técnicas para lograr una construcción conjunta del conocimiento. Entre dichas técnicas encontramos:

a) preguntas directas, o mediante fornece pistas para obtener conocimiento relevante de los alumnos;

b) confirmaciones, rechazos, repeticiones, elaboraciones y reformulaciones, que sirven como respuesta del tutor hacia los estudiantes;

c) frases del tipo nosotros, recapitulaciones literales y reconstructivas para recuperar experiencias compartidas con los alumnos; 
d) realizar afirmaciones abiertas o provocativas que generen réplicas o desacuerdos, invitar a elaborar, admitir perplejidades, animar a que los alumnos hagan preguntas con el objetivo de comenzar o alargar discusiones en el foro.

\section{A MODO DE CONCLUSIÓN}

Dado que las nuevas tecnologías cada vez cobran mayor relevancia para las oportunidades educativas y la participación en las áreas social, económica, política y cultural, quedar excluido de ellas implicaría una seria restricción a las posibilidades de vida académica. Tener acceso no significa sólo contar con una forma de utilizar computadoras y una conexión a Internet. El acceso debe ser reconsiderado como un objetivo social más complejo.

A través de diferentes apreciaciones que nos ofrecen tanto estudiantes y tutores, ambos remarcando su participación en la sección Foro, podemos concluir observando aquellos rasgos de las tecnologías que perjudican y otros que facilitan el proceso de enseñanza y aprendizaje.

Al dialogar con el tutor sobre las facilidades que se pueden presentar con el uso de las TICs al enseñar la asignatura contable a distancia, respondió que:

a) posibilita establecer una comunicación con sus alumnos en cualquier momento del día;

b) permite realizar un seguimiento del proceso de aprendizaje en un contexto masivo;

c) facilita el manejo de otros contenidos, más allá de la bibliografía planteada;

d) amplía la posibilidad de mostrar nuevos métodos que agilizen la resolución de problemas y visualizar la aplicación de algunos contenidos con software vinculados a problemáticas contables;

e) el crecimiento epistemológico en la selección del contenido a enseñar;

f) la elaboración de materiales de estudio específicos para esta modalidad.

Asimismo, al responder acerca de las obstrucciones que le proporcionaron las TICs en la enseñanza a distancia, hizo referencia a:

a) dificultades en los aspectos técnicos de utilización de la plataforma y la red;

b) la disponibilidad de tiempo requerida por el tutor fuera del horario habitual de clases;

c) la falta de capacitación pedagógica / didáctica.

Podemos inferir de los resultados anteriores que es indispensable adquirir habilidades y conocimientos acerca del funcionamiento de los medios tecnológicos para hacer un correcto y efectivo uso real de sus atributos. De no ocurrir esta situación, se generarían dificultades de acceso a las tecnologías y al conocimiento de la materia. Asimismo, el tutor argumenta que la producción de materiales educativos lo lleva a la discusión en profundidad de los contenidos a ser enseñados y lo obliga a mantener el estado del arte de la asignatura contable tratada.

En nuestro intento de identificar los atributos de los medios en educación a distancia, podemos observar que el Foro es, en particular, un medio donde los entrevistados expresan encontrar la utilidad de un intercambio activo y que la discusión y elaboración de materiales educativos los llevó a asumir una nueva mirada de la contabilidad. Este nuevo enfoque, implicó una rediscusión de los contenidos a ser enseñados, asumiendo un modelo de enseñaza más crítico y reflexivo.

A modo de cierre y retomando la pregunta formulada sobre la incidencia de las TICs en la enseñanza, podríamos decir que su uso potenciará los buenas prácticas de enseñanza en aquellos casos donde la tecnología puede estar integrada a la propuesta metodológica de manera tal que favorezca la construcción del conocimiento. Por el contrario, el abuso o el uso de las mismas mediante un empelo banal o superficial y que parte de una concepción meramente utilitarista, seguramente producirá efectos totalmente contrarios a los deseados. 


\section{REFERENCIAS}

ANDREONE, A. Un análisis focal de las experiencias vividas por alumnos y profesores de Cátedras que utilizan la TICs. Córdoba, Argentina: Proyecto SeCyT, 2002.

AUSUBEL, D. Psicología educativa: un punto de vista cognitivo. México, Trillas, 1976.

BURBUlES, N.; CALLISTER, T. Educación: riesgos y promesas de las nuevas tecnologías de la información. Buenos Aires: Ed. Granica, 2001.

GOLDENHERSH, H. et al. Deserción estudiantil en la universidad: el caso de la Faculad de Ciencias Económicas - FAC. Córdoba: Asoc. Coop. de la Facultad de Ciencias Económicas de la UNC, 2006.

JACKSON, P. et al. Practica de la enseñanza. Buenos Aries: Amorrortu editores, 2002.

LITWIN, E. Las configuraciones didácticas: una nueva agenda para la enseñanza superior. Buenos Aires: Ed. Paidos, 1997.

LITWIN, E. Tecnologías educativas en tiempos de Internet. Compilación. Buenos Aires: Ed. Amorrortu, 2005.

MAGGIO, Mariana. Educación a distancia en los 90. En: LITWIN, E. et al. (Coord.). El tutor en educación a distancia: compilación. Buenos Aires: Ed. Amorrortu, 2006.

MERCER, N.; GONZÁLEZ ESTEPA, F. La educación a distancia, el conocimiento compartido y la creación de una comunidad de discurso internacional. En: LITWIN, E.; LIBEDINSKY, M. La educación a distancia: deseos y realidades. Buenos Aires, Argentina, OEA, 1991.

SELTZER, J.C. Docencia y contabilidad. Revista Iberoamericana de Educación de la Organización de Estados Iberoamericanos, 2004. Disponible en: (http://www.rieoei.org/deloslectores/711Seltzer.PDF>. Aceso en: 26 enero 2009.

SILVA PALAVECINOS, B. Desde el proceso primario: un enfoque para una comprensión más eficiente de la dualidad económica, el fenómeno contable. Tesis (Magíster en Gestión, mención Contabilidad y Finanzas) - Pontificia Universidad Católica de Valparaíso, Valparaíso, Chile, 2003.

TUA PEREDA, J. En torno a la docencia de la Contabilidad: una reflexión personal. Revista Técnica Contable, v. 47, n. 557, p. 313-334, 1995.

ROJAS ROJAS, W. Congoja por una ecuación contable fútil. Revista Contaduría Universidad de Antioquia, Medellín, n. 52, p. 269, enero-junio, 2008. 